\title{
Representation of the process of sudden outbursts of coal and gas using a knowledge graph
}

\author{
Irina Osipova ${ }^{1 *}$, Veselina Gospodinova ${ }^{2}$ \\ ${ }^{1}$ Senior researcher, Candidate of engineering science. Institute of Mining, The Ural Branch of the \\ Russian Academy of Sciences. 620075, Yekaterinburg, Russia. \\ ${ }^{2}$ Mining and Geological University «St. Ivan Rilskiy» Mining and geological faculty, department" \\ Mine-surveying and geodesy "1700, Sofia, Bulgaria
}

\begin{abstract}
In the process of developing a coal deposit, significant amounts of data and extensive knowledge about the object of operation are accumulated. This data and knowledge may not be structured, not reliable, contradictory. In turn, for the further conduct of the entire complex of mining operations and the development of a coal mining enterprise, structured and reliable data and knowledge are needed. The aim of the study is to propose structuring knowledge about the process of sudden outbursts of coal and gas by presenting knowledge about the subject of research in the form of an elementary knowledge graph. The study is based on an ontological approach to solving the issue of safe mining, namely, to study the problem of sudden emissions of coal and gas from the standpoint of the fact that the release is considered before it has occurred. To solve the existing problem, it is proposed to create an elementary knowledge graph that takes into account geological, hydrogeological, geophysical, mining information about the subsoil use object, as well as geomechanical and geodynamic processes, and physicochemical mass transfer processes occurring in the coal seam and the accumulated experience and knowledge of miners using methods Data mining. As a result of the study, we can conclude that it is necessary to create a network of elementary knowledge graphs, and use other methods of knowledge extraction. For further analysis and refinement of data and knowledge about the process of sudden coal and gas emissions. Key words: outburst, coal, gas, intelligent control, knowledge graph.
\end{abstract}

\section{Introduction}

In the process of developing a coal deposit, significant amounts of data and extensive knowledge about the object of operation are accumulated. This data and knowledge may not be structured, not reliable, contradictory. In turn, structured and reliable data and knowledge are needed for the further conduct of the entire range of mining operations and the development of a mining enterprise. Note the following that here we are talking about intelligent management [1] of a mining enterprise (in this case it is understood as a complex

\footnotetext{
*Corresponding authors: minesur@mail.ru
} 
system [2]), with all standard and non-standard situations, with formalized and unformalized data and knowledge, incomplete, heterogeneous, contradictory information and data on the subsoil use process. [3]

Note that we can judge about the organization of data accumulation during the development of a coal deposit only on the basis of existing mining and geological, mining and technological, geophysical documentation or reporting documentation on mining and geological and other types of work included in the GIS system, as well as thanks to the experience and knowledge of qualified employees and workers. Thus, huge amounts of data appear, where it is necessary to distinguish between the collected data in terms of consistency, quality and reliability.

The accumulated vast amounts of data for the entire period of operation of a coal deposit may not be known to the chief engineer of a coal mining enterprise for various reasons. One of them is the complexity of information retrieval (this also applies to the GIS system used at the enterprise), analysis and interpretation of data. Therefore, for example, the need to own a picture of the information field (information, data and knowledge) about all mining facilities of a coal deposit is one of the main tasks for the chief engineer and making decisions on its safe and without emergency mining and operation. Since one of the geodynamic phenomena that most often occurs during the development of a coal seam are sudden emissions of coal and gas. Sudden outbursts of coal and gas pose the greatest threat among other dynamic phenomena. Their manifestation is especially dangerous in the powerful and very gas-bearing strata of the Kuznetsk Basin, when, with the sudden destruction of the massif, huge volumes of gas are released and spread through the mine workings, which can lead and more than once led to disasters. [4] Thus, there is a need to predict and prevent this gas-dynamic phenomenon.

The emerging need for structuring data and knowledge, namely, in the transition from a huge array of data to the creation of «valuable» and «useful» data and knowledge, will make it possible to create a reliable and objective picture of the development and operation of a coal deposit in order to solve one of the main tasks facing the management of a coal mining enterprises to predict and prevent sudden emissions of coal and gas.

In this regard, the aim of the research is to propose structuring knowledge about the process of sudden outbursts of coal and gas by presenting knowledge about the subject of research in the form of an elementary graph of knowledge. The task of the work is to show that within the framework of the ontological approach, it is possible to describe the object of research of a complex structure and the characteristic relationships between them. In the framework of the geomechanical approach, we will be guided by the proposal of Corresponding Member of the Russian Academy of Sciences G. I. Gritsko [4] to study a sudden release of coal and gas before the release did not occur.

\section{Research theory}

Currently existing approaches to processing huge data sets, graph databases such as NOSQL [6], and ontologies (knowledge graph) are aimed at solving multidimensional problems arising in the intellectual management of a mining corporation.

It should be noted here the following NOSQL database graphs provide efficient access to data, most suitable for the execution of specific algorithms [7].

In turn, knowledge graphs, analyzing a multitude of disparate data sources within a company [8] containing graph storages of semantic metadata (or knowledge formalized using special formal semantic languages) ... act as a semi-structured conceptual schema of the subject area [9]. There are other opinions regarding the understanding and representation of knowledge graphs, for example, according to the authors [10], the knowledge graph is a research method that combines the theory and method of applied 
mathematics, graphics, information visualization technologies, ... It shows the dynamic development of knowledge and complex knowledge based on data mining, information processing, knowledge assessment and graphical rendering. According to the authors, [11] the knowledge graph is also known as a scientific knowledge graph, domain knowledge visualization and knowledge domain maps. It is a series of different graphs that show the development of scientific knowledge and structural relationships.

In this regard, we can conclude that the knowledge graph allows you to visually represent formalized and non-formalized (complex) knowledge in the form of a conceptual scheme of the subject area, to assess this knowledge using various methods of applied mathematics and data mining.

\section{Results of work}

For a visual representation of an elementary graph of knowledge about the process of a sudden release of coal and gas. It is necessary to determine what data and knowledge the coal mining enterprise possesses. To represent the hierarchy or sequence of coherence of this existing set of information.

At the initial stage, it is necessary to determine what concepts and terminology a coal mine operates in order to understand the process of a sudden release of coal and gas. For this, a classification dictionary (thesaurus) is compiled. It is then compared with internationally accepted definitions of the sudden outburst of coal and gas. If there are no contradictions and inconsistencies, then the dictionary is formalized by creating a semantic network. Further, the geological, hydrogeological, geophysical, mining documentation that exists in the GIS system is analyzed, which gives an idea of the subsoil use object, in particular, about each worked-out coal seam and put into development. Data analysis is carried out using Data mining methods, which allow [12] to detect various deviations, cluster data, create various classifications. The extracted knowledge is recorded in the elementary knowledge graph.

Then the geomechanical, geodynamic and physicochemical mass transfer processes in multiphase coal seams of different stages of metamorphism are analyzed during their development at specified depths and temperature background [13]. At this stage, vast amounts of information, data and knowledge appear, which may be contradictory, unreliable, inconsistent, and unclear in the understanding of the ongoing processes. Here, in addition to data mining methods, the knowledge of miners (mining engineers directly involved in coal mining) will be needed. For this, there will be a need to create additional knowledge graphs within the elementary knowledge graph that characterize each process and illustrate knowledge about a sudden release of coal and gas.

The knowledge gained from the miners is interpreted in the classification. Knowledge that cannot be classified, knowledge clusters are created for them, combining by a certain property of information or indicators. Subsequently, they can turn into either «useful» knowledge or non-interpretable data.

As a result, we obtain an elementary knowledge graph (see Fig. 1) with the following fields (classes) about the process of a sudden outburst of coal and gas:

-Classification dictionary (thesaurus);

-Geological information about the subsoil use object;

-Hydrogeological information about the subsoil use object;

-Geophysical information about the subsoil use object;

-Mining technical information about the subsoil use object;

-Geomechanical processes occurring in the coal seam;

-Geodynamic processes occurring in the coal seam;

-Physical and chemical mass transfer processes occurring in the coal seam; 
-Practical knowledge of specialists in coal mining.

In fig. 1 presents an elementary graph of knowledge characterizing the process of a sudden outburst of coal and gas from the standpoint of an ontological approach and taking into account the peculiarity of the study that the process is studied before the outburst has not occurred.

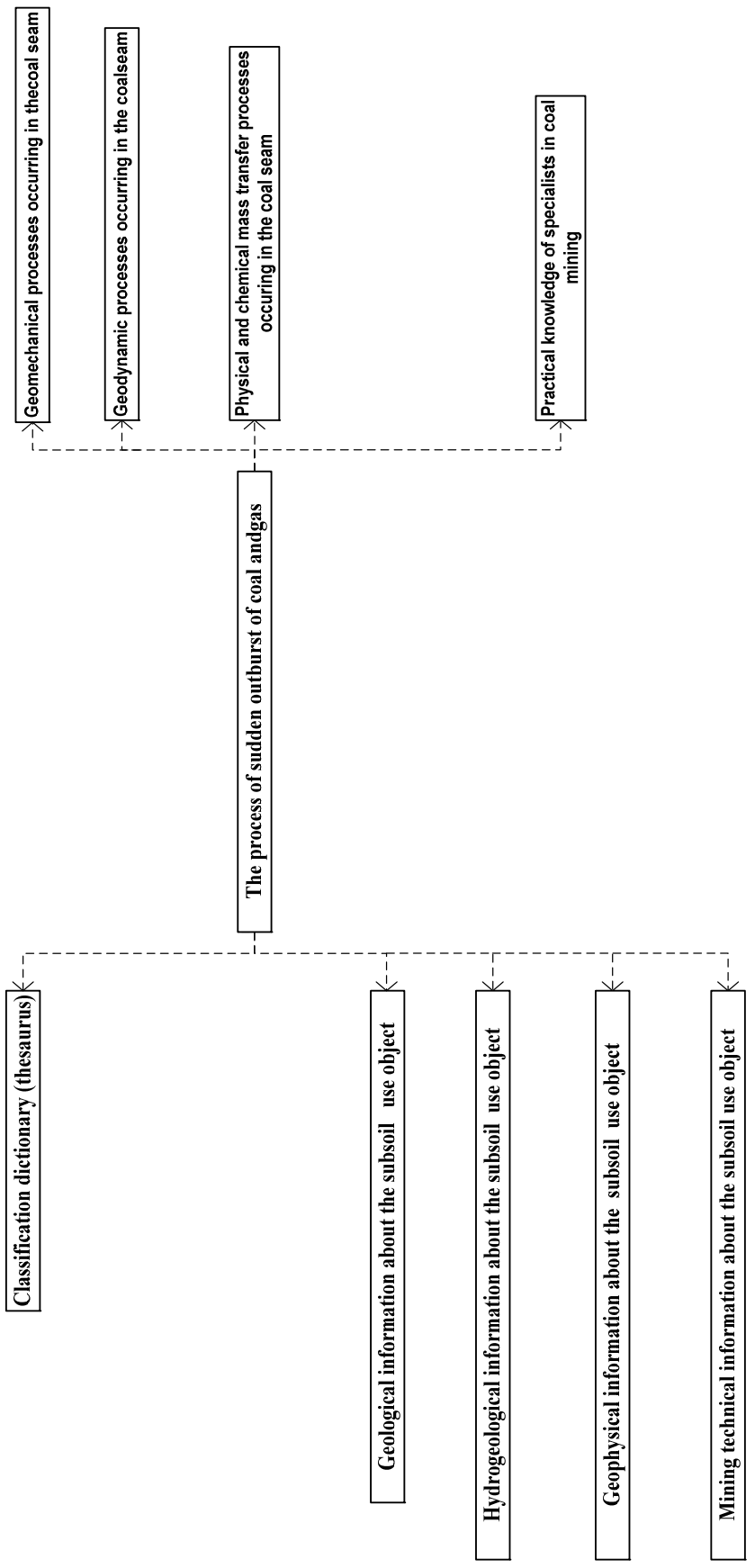

Fig. 1. Representation of the process of a sudden outburst of coal and gas using the knowledge graph 


\section{Conclusions}

In this paper, we propose an approach to creating an elementary graph of knowledge about the process of sudden coal and gas outbursts from the standpoint of an ontological approach and fulfilling the recommendation of Corresponding Member of the Russian Academy of Sciences G.I. Research is complicated by the large amount of data and extensive knowledge about the object of operation. In addition, visualization of the process of a sudden outburst of coal and gas can be contradictory, since the data and knowledge themselves may not be reliable, subjective and contradictory.

The obtained elementary graph of knowledge will make it possible to form an idea of a sudden outburst of coal and gas and conduct further research in the framework of a more detailed study of the geomechanical and geodynamic processes of this dynamic phenomenon, taking into account the accepted restrictions and classifying genetically different types of outwardly similar phenomena using the ontological approach.

The work was carried out within the framework of the State assignment, topic No. 0328-2019-0005 (2019-2021).

\section{List of references}

1.

I. A. Osipova, GIAB, 11(37) (2019). DOI: 10.25018/0236-1493-2019-11-37-551558

2. M. Yu. Okhtilev, B. V. Sokolov, R. M. Yusupov, Intellectual technologies for monitoring and control of the structural dynamics of complex technical objects (2006)

3. I. A. Osipova, Mat. International. scientific and technical conf. dec. eco-friendly and technological. samples. horn. manuf. on the territory. Russia, Blizh. and Dal. Zarubezh., 179 (2019)

4. V. S. Zykov, I. L. Abramov, D. V. Torgunakov, GIAB, 6, 319 (2013)

5. $\quad$ G.I. Gritsko URL: http: //www.nsc.ru/HBC/article.phtml?nid $=428$ \& id $=17$, (2007) (date accessed: 30.10.2019)

6. I. Robinson, J. Webber, E. Eifrem Graph Databases: New Opportunities for Connected Data O'REILLY (2015)

7. E. Khlyzov URL: http://osp.ru/os/2018/03/13054405, (2018) (date accessed: 29.07.2020)

8. Hubauer T. et al. (P\&D/Industry/Blue Sky) (2018)

9. D. Muromtsev, A. Romanov, D. Volchek Cont. Eng. Russia, 5(83), 32 (2019)

10. Y. Zhu, W. Zhou, Y. Xu, J. Liu, Y. Tan Hindawi Scient. Program. 2-3 (2017). DOI 10.1155/2017/5072427

11. Q. Liu, Y. Li, H. Duan, Y. Liu, Z. Qin Comp, Res. \& Devel, 53(3) (2016)

12. F. Gorunescu, Data Mining Concepts, Models and Techniques, (2011). DOI 10.1007/978-3-642-19721-5

13. V.N. Oparin, T.A. Kiryaeva, West Engineering School FEFU, 3 (20) (2014) 